\title{
Improving Throughput and QoS with IEEE 802.11e
}

\author{
Takumi Sanada, Xuejun Tian, Takashi Okuda, and Tetsuo Ideguchi
}

\begin{abstract}
WLANs have become increasingly popular and widely deployed. The MAC protocol is one of the important technology of the WLAN and affects communication efficiency directly. In this paper, focusing on MAC protocol, we propose a novel protocol that network nodes dynamically optimize their backoff process to achieve high throughput while supporting satisfied QoS. The total throughput decreases heavily and cannot guarantee QoS under a high traffic load, which needs to be improved. Through theoretical analysis, we find that the average idle interval can represent current network traffic load and can be used together with estimated number of nodes for setting optimal $C W$. Since necessary indexes can be obtained through direct measurement from channel, our scheme will not increase any added load to networks. Through simulation comparison with conventional method, we show that our scheme can greatly enhance the throughput and the QoS no matter the network is in saturated or non-saturated case, while maintaining good fairness.
\end{abstract}

Index Terms-WLANs, MAC, EDCA, throughput.

\section{INTRODUCTION}

Wireless local area networks (WLANs) have become increasingly popular and widely deployed. In two channel access methods DCF and an optional centralized PCF, due to inherent simplicity and flexibility, the DCF is preferred in the case of no base station such as vehicle to vehicle communications. Since all the nodes share a common wireless channel with limited bandwidth in the WLAN, it is highly desirable that an efficient and fair medium access control (MAC) scheme is employed. However, for the IEEE 802.11 DCF, there is much room for improvement in terms of both efficiency and fairness. Cali et al. pointed out in [1] that depending on the network configuration, DCF may deliver a much lower throughput compared to the theoretical throughput limit. Meanwhile, as demonstrated in [2], the fairness as well as throughput of the IEEE 802.11 DCF could significantly deteriorate when the number of nodes increases.

Although extensive research has been conducted to improve throughput ([1], [3]-[8]) or fairness ([6], [7]), except in ([6], [7]), these two performance indexes are rarely considered together. In DCF, estimating the number of nodes is difficult due to that each node can enter or leave the network freely. And these schemes avoid to estimate the number of nodes. In [9], the number of nodes is estimated,

Manuscript received November 12, 2014; revised April 10, 2015. This work was supported by the Ministry of Education, Culture, Sports, Science and Technology, Grant-in-Aid for Scientific Research (C), 24500088 and 24500087, 2014.

The authors are with the School of Information Science and Technology, Aichi Prefectural University, Japan (e-mail: im141008@cis.aichi-pu.ac.jp, tan@ist.aichi-pu.ac.jp, ideguchi@ist.aichi-pu.ac.jp). however, it is complicated, takes time for estimating number of nodes and actual number of nodes maybe have changed. In [7] and [10], these schemes observe the average idle interval, adjust the $C W$ in order to obtain higher throughput. These schemes do not estimate the number of nodes and have an issue which the variation in $C W$ of each node is large, which results in fairness degradation. In [11], based on [7], to improve the problem of fairness which is important for real time communication, authors introduced a method to achieve better fairness but the improvement is not enough. In [12], authors proposed a novel protocol by observing the channel event to estimate the number of nodes by using the channel event probability, and tunes the network to obtain high throughput with good fairness according to the number of nodes. This is proved to be effective. In the other hand, how to support QoS in DCF is important and difficult. Though, IEEE 802.11e EDCA support QoS for traffics with different priorities. A node of EDCA can control its own traffic according to their priorities but cannot achieve high throughput and good QoS simultaneously, especially when each node have different traffic with different priorities. Therefore, in this paper, we use the method in [12] to tune $C W$ according to each priority to achieve good performance both on throughput and QoS. The related works [13]-[16] proposed the scheme that improves both throughput and QoS in EDCA, but did not take fairness into account and estimate the number of nodes. In this paper, we aim to enhance throughput, fairness and QoS for EDCA at the same time by solving the problems of conventional method and estimating the number of nodes briefly and dynamically. And we propose a novel MAC scheme that Optimizing Backoff with QoS, named as OBQ.

The remainder of this paper is organized as follows. In Section II, we analyze the conventional method and problems. We elaborate on our key idea and the theoretical analysis for improvement in Section III. Then we present our proposal OBQ scheme in detail. Section IV gives performance evaluation and the discussions on the simulation results. Finally, concluding remarks are given in Section V.

\section{COnVEntional Method}

\section{A. Distributed Coordination Function (DCF)}

The IEEE 802.11 DCF is based on a mechanism called carrier sense multiple access with collision avoidance (CSMA/CA). In DCF, a node with a packet to transmit initializes a backoff timer with a random value selected uniformly from the range $[0, C W-1]$, where $C W$ is the contention window in terms of time slots. After a node senses that the channel is idle for an interval called DIFS (DCF inter-frame space), it begins to decrease the backoff timer by 
one for each idle time slot. When the channel becomes busy due to other nodes' transmissions, the node freezes its backoff timer until the channel is sensed idle for another DIFS. When the backoff timer reaches zero, the node begins to transmit. If the transmission is successful, the receiver sends back an acknowledgment (ACK) after an interval called SIFS (short inter-frame space). Then, the transmitter resets its $C W$ to $C W \mathrm{~min}$. In case of collisions, the transmitter fail to receive the ACK from its intended receiver within a specified period, it doubles its $C W$ until reaching a maximum value $C W \max$ after an interval called EIFS (extended inter-frame space), chooses a new backoff timer, and start the above process again. When the transmission of a packet fails for a maximum number of times, the packet is dropped.

\section{B. Enhanced Distributed Channel Access (EDCA)}

In IEEE 802.11e, hybrid coordination function (HCF) is defined as the MAC scheme. It includes EDCA and contention-free HCF controlled channel access (HCCA) to provide QoS stations (QSTAs) with prioritized access. EDCA is based on CSMA/CA and extends DCF by means of the similar parameters that are used to access the channel. In EDCA, nodes have four $A C, A C[V O]$ (voice), $A C[V I]$ (video), $A C[B E]$ (best effort) and $A C[B K]$ (background), where $A C$ $[V O]$ is the highest priority while $A C[B K]$ is the lowest priority and each $A C$ is assigned by the kind of packet to transmit. Each $A C$ behaves like a virtual station which contends for access to the medium and starts its backoff independently. When a collision occurs among different $A C \mathrm{~s}$ of the same station, i.e., two backoff counters of $A C$ s reaches zero at the same time, the packet of the highest priority $A C$ is transmitted while the lower priority $A C$ performs backoff again as if a collision occurred. In each $A C$, there is arbitration inter frame space (AIFS) instead of DIFS, $C W \min , C W \max$ and transmission opportunity (TXOP), respectively. TXOP means that a node transmits multiple packets as long as the duration of the transmissions does not extend beyond TXOP.

\section{Problems}

There are three problems in IEEE802.11 MAC. First, throughput decreases in case of increasing the number of nodes. A collision occurs when two or more nodes start transmitting at the same time. Generally, in WLANs, node cannot detect a collision during transmission. Node continues transmitting until completing transmission even if a collision occurs. Nodes around transmitter are also affected and waste limited bandwidth. When a collision is occurred the $C W$ is doubled and a new backoff procedure is started. In theoretical analysis, we can obtain high throughput by using optimal $C W$ according to the number of nodes. And the larger the number of nodes, the larger optimal $C W$ in order to avoid a collision. In conventional method, since the $C W$ is reset to $C W \min$ in case of transmission is successful, the number of collisions increases according to the number of nodes and throughput decreases.

Second, the variation of $C W$ is large. It means that the variation of the transmission delay is large. Also, the jitter is large and the fairness decreases. To solving these two problems, all nodes always have the $C W$ around the optimal $C W$ according to the number of nodes.
Finally, QoS is not guaranteed enough. Since QoS is supported in IEEE $802.11 \mathrm{e}$, the high priority $A C$ transmits with priority and needs to act as the high guarantee of successful transmission. However, QoS becomes low in the case of the number of nodes increasing [17], [18]. In this paper, we can solve these problems and enhance both of the throughput and the QoS.

\section{ANALYSIS AND THE PROPOSAL OF OPTIMIZING BACKOFF BY DYNAMICALLY ESTIMATING NUMBER OF NODES}

\section{A. Optimal Backoff}

In the IEEE 802.11 MAC, an appropriate $C W$ is the key to providing throughput, fairness and QoS. In [1], the IEEE 802.11 DCF is analyzed based on an assumption that, in each time slot, each node contends for the medium with the same probability $p$ subject to $p=1 /(E[B]+1)$, where $E[B]$ is the average backoff timer and equals $(E[C W]-1) / 2$ for DCF. Since our OBQ would enable all the nodes to settle on a quasistable $C W$ shortly after the network is put into operation, for simplicity we assume that all the nodes use the same and fixed $C W$. Consequently, we have

$$
p=\frac{2}{C W+1}
$$

As all the expectation signs $E$ can be removed. Channel events can be thought as three types of events, successful transmission, collision, idle. Suppose every node is an active one, i.e., always having packets to transmit. For every packet transmission, the initial backoff timer is uniformly selected from $[0, C W-1]$. For each virtual backoff time slot, it may be idle, busy due to a successful transmission, busy due to collision. Accordingly, we denote by $p_{i d l}, p_{s}$, and $p_{c o l}$ the probabilities of the three types of events, respectively. Thus, we can express the above probabilities as follows,

$$
\begin{aligned}
& P_{i d l}=(1-p)^{n} \\
& P_{s}=n p(1-p)^{(n-1)} \\
& P_{c o l}=1-P_{i d l}-P_{s}
\end{aligned}
$$

where $n$ is the number of active nodes. In the following, we give the method to estimate the number of node on line by three parameters $p_{i d l}, p_{s}$, and $p_{c o l}$ which can be obtained directly by listening channel for a certain interval. Then, using obtained $p_{i d l}, p_{s}$, and $p_{c o l}$, we give the method to maximize the throughput dynamically. Calculating number of nodes directly by (2) is inefficient and realistic. Here, we use a simple and effective method which is suitable for real time estimating. From (2), we have $p_{i d l} / p_{s}=(1-p) /(n p)$, then $p=$ $p_{s} /\left(n p_{i d l}+p_{s}\right)$. Substitute $p$ in $p_{i d l}=(1-p)^{n}$, it becomes as following,

$$
P_{i d l}=\left(1-\frac{P_{s}}{n P_{i d l}+P_{s}}\right)^{n} .
$$


Let $f_{i d l}(n)=\left(1-\frac{P_{s}}{n P_{i d l}+P_{s}}\right)^{n}$ where $p_{i d l,} p_{s}$ and $p_{c o l}$ are known parameters and $n$ is unknown parameter that needs to be estimated. Then when $f_{i d l}\left(n_{0}\right)=p_{i d l}, n_{0}$ is the needed value. Before giving estimation algorithm, we show the following lemma.

Lemma : $f_{\text {idl }}(n)$ is monotone function

Proof: $d f / d n=\left[\ln \left(1-P_{s} /\left(n P_{i d l}+P_{s}\right)\right)+P_{s} /\left(n P_{i d l}+P_{s}\right)\right]\left(1-P_{s}\right.$ $\left./\left(n P_{i d l}+P_{s}\right)\right)^{n}$. It can be found that the second term is always plus. Let $x=P_{s} /\left(n P_{i d l}+P_{s}\right)$, then $0 \leq x \leq 1$. Then, the first term of $d f / d n$ becomes $\ln (1-x)+x$ which changes from 0 to $-\infty$ when $x$ changes from 0 to 1 . So, it can be understood that $d f / d n$ is not plus.

We can estimate the number of nodes by the simple calculation method, without solving a complicated equation using this characteristic. As shown in Fig. 1, the monotone function $f_{\text {idl }}(n)$ always decreases as number of nodes increasing. Since $P_{i d l}$ is a known value, $f_{i d l}(n)$ should be adjusted in agreement with $P_{i d l}$. When $P_{i d l}$ is equal to $f_{i d l}(n), n$ is the number of nodes deployed in real network.

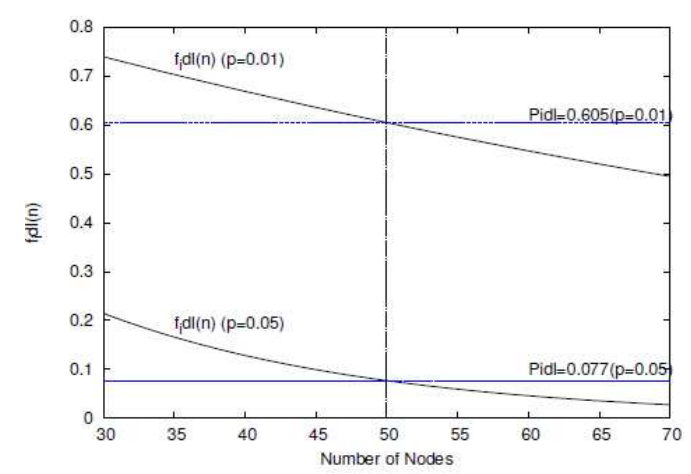

Fig. 1. Monotone function $f_{\text {idl }}(n)$ when real value of $n$ is 50 .

The above character is favorable for estimating number of nodes $n$ which can be calculated by the following dichotomy algorithm. Supposing $n$ is in a range $\left[0, n_{\max }\right]$, initially let $n_{\text {try } 1}$ $=n_{\max } / 2$ and substitute it into $f_{\text {idl }}(n)$. Then compare $f_{\text {idl }}\left(n_{\text {try } 1}\right)$ with $p_{\text {idl }}$. If $f_{\text {idl }}\left(n_{\text {try } 1}\right)>p_{\text {idl }}$, we should set $n_{\text {try } 2}=\left[n_{\text {try } 1}+n_{\text {max }}\right] / 2$. Otherwise, we should set $n_{\text {try } 2}=\left[n_{\text {try } 1}+0\right] / 2$ for the following calculation. Obviously, this method is simple and effect. For example, when $n_{\max }=100$, we just need calculate four times to estimate $n$ in the worst case with maximum error 3 . In the following, we give the method how to dynamically tune $C W$ to enhance throughput, fairness and QoS.

In [12], as a condition that the throughput reaches to the maximum, the relation between $C W$ and the number of nodes is analyzed as follows:

$$
L_{i d l}=\frac{C W-1}{2 n}
$$

where $L_{i d l}$ is the average idle interval and a constant which can be thought as independent of the number of nodes and frame-length approximately. From above (4), we can obtain the optimal $C W$ that $C W=2 n L_{i d l}+1$ using estimated the number of active nodes. The $C W$ is a linear function of the number of nodes. Since we are interested in tuning the network to work with maximal throughput, given the linear relationship, we can achieve this goal by adjusting the size of $C W$. In other words, each node can estimate the number of nodes and adjust its backoff window accordingly such that the total throughput of the network is maximized.

\section{B. Enhancement of $Q o S$}

In above section, we introduced a method to maximum total throughput under the condition that all nodes are in the saturation status and the same situation. Here, we use this method to improve EDCA. For IEEE 802.11e, each node has four $A C \mathrm{~s}, A C[V O], A C[V I], A C[B E]$ and $A C[B K]$. Because $A C[B K]$ is close to $A C[B E]$, we take $A C$ s as $A C[V O], A C[V I]$ and $A C[B E]$ in the analysis. Each node of EDCA can set optimal $C W$ according to the number of nodes by equation (4) called $C W_{o p}$ as shown above. It is well known that the throughput of each $A C$ in the same node is inversely proportional to its $C W \mathrm{~s}$ that $C W[V O], C W[V I]$ and $C W[B E]$. So if knowing the optimal $C W_{o p}$ for a node, we can set optimal $C W$ for each $A C$ and the total throughput of the node is equal to the sum throughput of all $A C$ s. In this case, there is a difference between IEEE 802.11 and IEEE 802.11e for using OBEN shown in [12]. In EDCA, each node is not always in the same situation that all $A C$ s of each node are saturated. However, this difference does not have serious influence, which can be understood by simulation results given in the following section. In EDCA, estimated the number of nodes can be thought in a wide sense. We assume $\rho_{V O}: \rho_{V I}: \rho_{B E}$ as the transmission opportunity of each $A C$. The rate of the transmission opportunity of each $A C$ can be expressed as

$$
\begin{aligned}
& \eta_{V O}=\frac{\rho_{V O}}{\rho_{V O}+\rho_{V I}+\rho_{B E}} \\
& \eta_{V I}=\frac{\rho_{V I}}{\rho_{V O}+\rho_{V I}+\rho_{B E}} \\
& \eta_{B E}=\frac{\rho_{B E}}{\rho_{V O}+\rho_{V I}+\rho_{B E}}
\end{aligned}
$$

Also, the attempt probability can be expressed as $p=$ $2 /\left(C W_{o p}+1\right)$ from (1). Considering from the attempt probability of a node, it becomes $p=p_{V O}+p_{V I}+p_{B E}$. When $C W$ increases, the attempt probability decreases, and moreover throughput also decreases. Approximately, throughput is in proportion to the attempt probability. From the above analysis, the attempt probability of each $A C$ can be expressed as, for example, $p_{V O}=\eta_{V O} \times p=\eta_{V O} \times 2 /\left(C W_{o p}+1\right)$. Consequently, $C W$ of each $A C$ can be expressed as following.

$$
\begin{aligned}
& C W[V O]=\frac{1}{\eta_{V O}} \times\left(C W_{o p}+1\right)-1 \\
& C W[V I]=\frac{1}{\eta_{V I}} \times\left(C W_{o p}+1\right)-1 \\
& C W[B E]=\frac{1}{\eta_{B E}} \times\left(C W_{o p}+1\right)-1
\end{aligned}
$$

Even when no nodes are the same state, namely, some nodes have traffic of a part of $A C$, the proposed method is 
effective. In this case, estimated number of nodes differs from a radical meaning. It becomes as a comprehensive index of network traffic. $C W$ of nodes that have traffic of a part of $A C$ may be larger than $C W_{o p}$. Nodes that have traffic of a part of $A C$ estimate the number of nodes smaller than actual nodes. Hence, the nodes adjust $C W$ around $C W_{o p}$, obtain high throughput. We prove it in Section IV. OBQ can offer QoS flexibly by the scheme how to adjust $C W$ of each $A C$ as shown above. According to the transmission opportunity of each $A C$, change the delay of each $A C$ but not change total throughput, OBQ can always maintain high throughput and provide the satisfied QoS.

\section{OBQ Scheme}

With (3), for estimating the number of nodes, we need to obtain $P_{i d l}, P_{s}$ and $P_{c o l}$. To obtain it, we can count the number of idle slots $\left(C_{i d l}\right)$, collisions $\left(C_{c o l}\right)$ and successful transmissions $\left(C_{s}\right)$ individually. To avoid occasional cases, $C_{i d l}, C_{c o l}$ and $C_{s}$ are expected to be measured in a certain period, for example a certain number of packet transmissions reaches a certain value. The $p_{i d l}, p_{s}$ and $p_{c o l}$ can be calculated as following:

$$
\begin{gathered}
P_{i d l}=\frac{C_{i d l}}{C_{i d l}+C_{s}+C_{c o l}} \\
P_{s}=\frac{C_{s}}{C_{i d l}+C_{s}+C_{c o l}} \\
P_{c o l}=\frac{C_{c o l}}{C_{i d l}+C_{s}+C_{c o l}}
\end{gathered}
$$

We can obtain the optimal $C W$ by (4) using estimated the number of active nodes. Then, each node can adjust its $C W$ dynamically hence tune the network to deliver high throughput. With obtained $C W$ and the transmission opportunity of each $A C, C W$ is set to each $A C$. According to the QoS requirement, $C W$ ratio in (6) can be set freely. In following, we give the tuning algorithm.

1) A node, say Node A, begins listening channel and counts events of idle slot, successful transmission and collision individually.

2) When Node A needs backoff and the number of packet transmissions reaches a certain number, calculates optimal $C W$ as new $C W$.

3) With the new $C W$ and the transmission opportunity of each $A C, C W$ is set to each $A C$, and then it returns to 1 ).

Ideally, each node should have the same $C W$ when the network enters into steady state in saturated case; in reality, each node set its $C W$ around the optimal $C W$. Using this scheme, high throughput, good fairness and satisfied QoS are achieved, which can be found in the following simulations.

\section{PERformance EVAluation}

In this section, we focus on evaluating the performance of our OBQ through simulations, which are carried out on OPNET Modeler [19]. For comparison purpose, we also present the simulation results for the IEEE 802.11e EDCA. IEEE $802.11 \mathrm{~g}$ is adopted as the wireless medium. The simulation parameters of IEEE802.11e are shown in Table I and the OBQ-specific parameters in Table II. In IEEE $802.11 \mathrm{e}$, sets the maximum $C W$ of each $A C$, but in OBQ, there is no upper bound of $C W$ of each $A C$. Not thinking a specific application, we assume network nodes are distributed at random in a round area with diameter of 200 meter and each node generates traffic according to a Poisson process with the same arrival rate. Each node selects a node in the center of a round area as a receiver. The arrival rate is kept increasing until the network is saturated. The target throughput ratio of each $A C$ should be set according to the QoS requirement. However, since the QoS requirement is not assumed in particular, all nodes have three $A C \mathrm{~s}$. The transmission opportunity of each $A C$ is set to $\rho_{V O}: \rho_{V I}: \rho_{B E}=15: 10: 1,60$ : $20: 1$, simulations are carried out in two patterns. For the approximation $1 / \rho_{V O}: 1 / \rho_{V I}: 1 / \rho_{B E}=C W[V O]: C W[V I]$ $C W[B E], C W$ is set to each $A C$. As shown below, OBQ exhibits a better performance.

\begin{tabular}{|c|c|}
\hline Parameter & Value \\
\hline Maximum number of nodes & 100 \\
\hline Lidl & 5 \\
\hline
\end{tabular}

\begin{tabular}{|c|c|}
\hline \multicolumn{2}{|c|}{ TABLE I: NETWORK CONFIGURATION } \\
\hline Parameter & Value \\
\hline SIFS & $10 \mu \mathrm{sec}$ \\
\hline Slot time & $20 \mu \mathrm{sec}$ \\
\hline EIFS & $364 \mu \mathrm{sec}$ \\
\hline AIFS[VO] & $50 \mu \mathrm{sec}$ \\
\hline AIFS[VI] & $50 \mu \mathrm{sec}$ \\
\hline AIFS[BE] & $70 \mu \mathrm{sec}$ \\
\hline TXOP[VO] & $1504 \mu \mathrm{sec}$ \\
\hline TXOP[VI] & $3008 \mu \mathrm{sec}$ \\
\hline TXOP[BE] & 0 \\
\hline MinCW[VO] MaxCW[VO] & $3 \sim 7$ \\
\hline MinCW[VI] MaxCW[VI] & $7 \sim 15$ \\
\hline MinCW[BE] MaxCW[BE] & $15 \sim 1023$ \\
\hline Max retry threshold & $256000 \mathrm{bits}$ \\
\hline Buffer size & $24 \mathrm{Mbps}$ \\
\hline Data rate & \\
\hline
\end{tabular}

TABLE II: BACKOFF PARAMETERS

\section{A. Estimating Number of Nodes}

OBQ can estimate number of contending nodes dynamically. Fig. 2 shows the estimated number of nodes of a node with condition of 50 nodes in offered load 1. From Fig. 2, we find that estimated number of nodes changes to big value close to 120 because all nodes begin to transmit at same time from the beginning of simulation and then converges to a comparatively stable value around 50 after a short time about $13 \mathrm{~s}$ which is related to algorithm of backoff parameters shown in Table III.

\section{B. Throughput}

First, since all nodes can obtain the almost same throughput, we present the total throughput of $A C[V O], A C[V I]$ and $A C[B E]$ obtained for the two schemes, i.e., OBQ and the IEEE 802.11e, under different offered load and packet sizes. Fig. 3 and Fig. 4 show the throughput results when the number of nodes is 50 and the packet sizes are 256, 640, 1280 and 1500 bytes, respectively. In figures, vertical axis expresses normalized throughput which is the ratio of actual throughput to network data rate (24Mbps) and horizontal axis expresses 
normalized offered total traffic. Note that the packet size is the size of payload data and does not include MAC overhead, which is one reason that the simulation results are lower than the theoretical value. In Fig. 3, we can find that when the traffic load is low, say lower than 0.3 , the throughput of OBQ with short packet size 256 bytes is similar to the IEEE 802.11 e but a little difference. The throughput is tiny more than offered load because of Poisson arrival used for packet generation. The throughputs of OBQ and the IEEE 802.11e begin to be lower than offered load, which mean packet loss. The throughputs of OBQ and the IEEE 802.11e increase with traffic load increase from 0.3 and reach saturation. The maximum throughput of $\mathrm{OBQ}$ is 0.32 which is higher than 0.26 of the IEEE $802.11 \mathrm{e}$ in the case of 256 bytes. Improvement reaches to $23 \%$. In the case of packet size 640 bytes, throughputs increase higher and the difference of OBQ and the IEEE 802.11e becomes bigger. In Fig. 4, the packet sizes are set as 1280 and 1500 bytes longer than above case. The same change tendency can be found like Fig. 4 . The improvement in throughput becomes higher in the case of longer packet size, which reaches about twice in the case of 1500 bytes packet size. Fig. 5 shows the total throughputs when the $C W$ ratio is changed. The $C W$ ratio has a little effect on the total throughputs performance.

Fig. 6 shows the saturation total throughputs with different packet sizes. Because the $C W$ ratio has little effect on the total throughput performance, Fig. 6 shows only the result of the $C W$ ratio $C W[V O]: C W[V I]: C W[B E]=2: 3: 30$ as one example. As shown in the figure, the throughput of OBQ rise when packet size increase and OBQ is not so sensitive to changes in the number of nodes because of optimized $C W$. In contrast, the throughput of IEEE 802.11e has little change when packet size is changed and becomes low as number of nodes increasing.

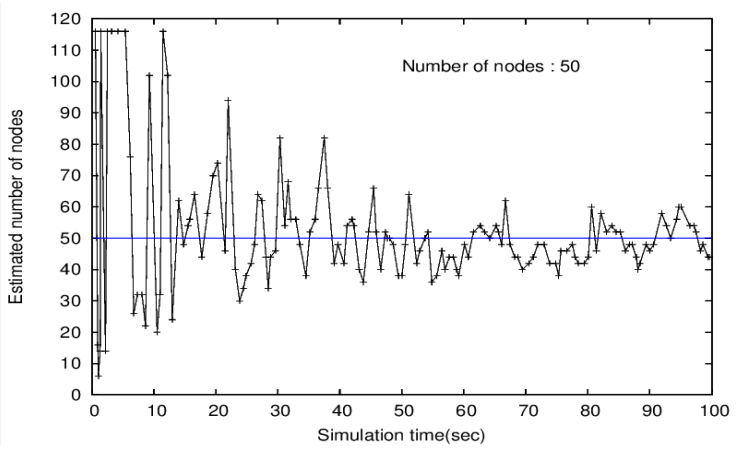

Fig. 2. Estimated number of nodes vs. simulation time.

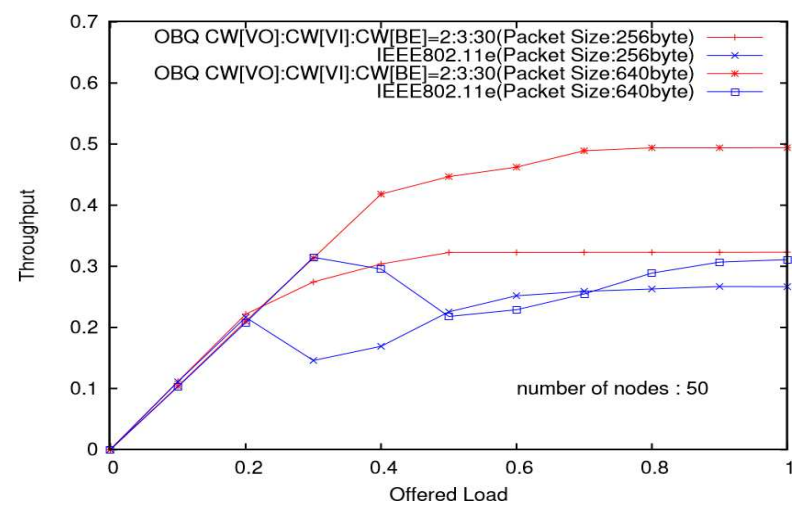

Fig. 3. Throughput of OBQ and IEEE 802.11e with different packet size.

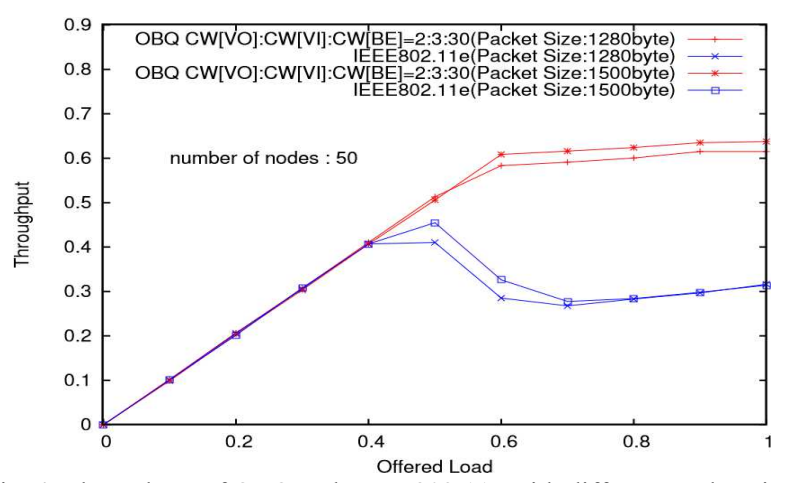

Fig. 4. Throughput of OBQ and IEEE 802.11e with different packet size.

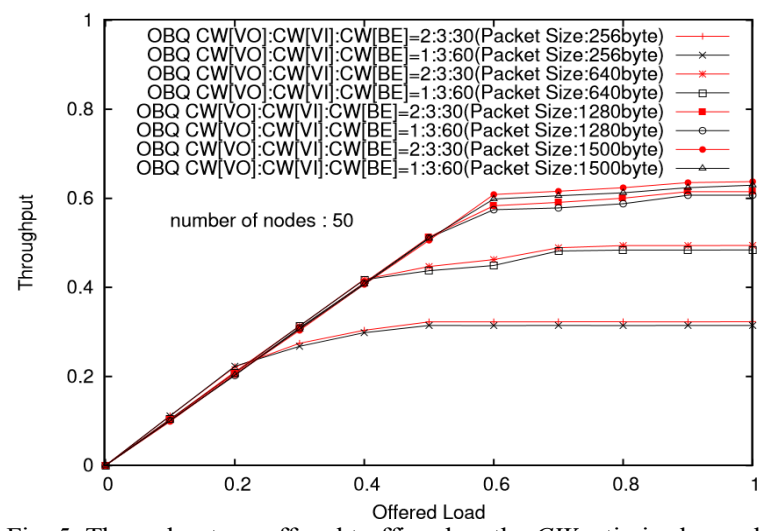

Fig. 5. Throughput vs. offered traffic when the $C W$ ratio is changed.

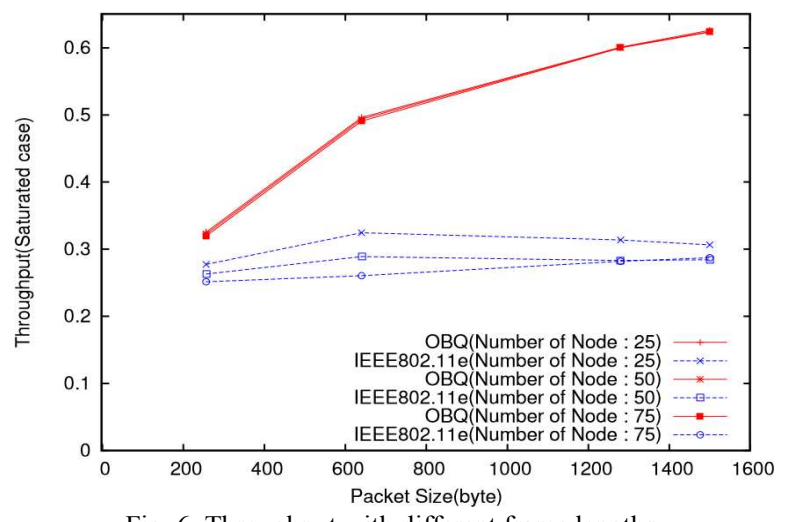

Fig. 6. Throughput with different frame lengths.

\section{Delay}

Fig. 7 and Fig. 8 show the delay results of each $A C$ when the number of nodes is 50 and the packet size is 1280 bytes since it is the same tendency even if packet size is changed. The delay is the time from head of the transmission queue to receiving ACK frame, does not include the time of queuing. Fig. 7 shows the delay results of $A C[V O]$ and $A C[V I$. When offered load is less than 0.6 , the delay of OBQ is lower than that of the IEEE 802.11e. However, from offered load is 0.6, the delay of OBQ is higher than that of the IEEE 802.11e. It is because that part of delay of IEEE 802.11e of dropped packets is ignored. Which does not mean the delay characteristics is good. We describe in detail in the next section of data dropped. Fig. 8 shows the delay of $A C[B E]$. The delay of OBQ is always lower that of the IEEE 802.11e. Fig. 9 and Fig. 10 show the delay results of each $A C$ when $C W$ ratio is changed. Fig. 9 shows the delay results of $A C[V O]$ and $A C[V I$. The delay is changed according to $C W$ ratio. Fig. 10 shows the delay result of $A C[B E]$. The same change tendency 
can be found like Fig. 9. Thus, OBQ changes the delay of each $A C$, does not change the total throughput when $C W$ ratio is changed.

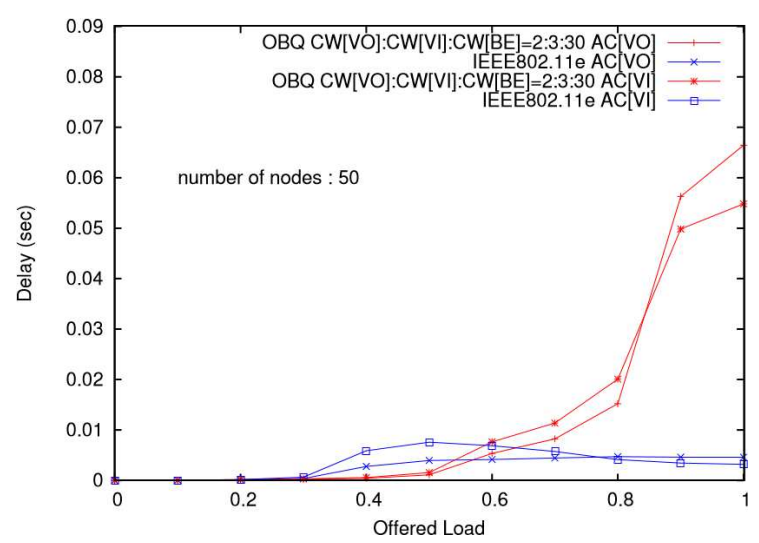

Fig. 7. Delay of $A C[V O]$ and $A C[V I]$ vs. offered traffic.

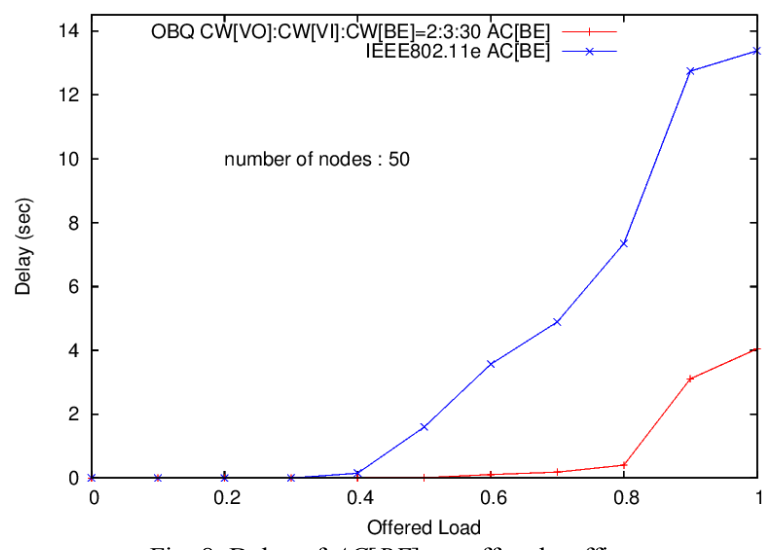

Fig. 8. Delay of $A C[B E]$ vs. offered traffic.

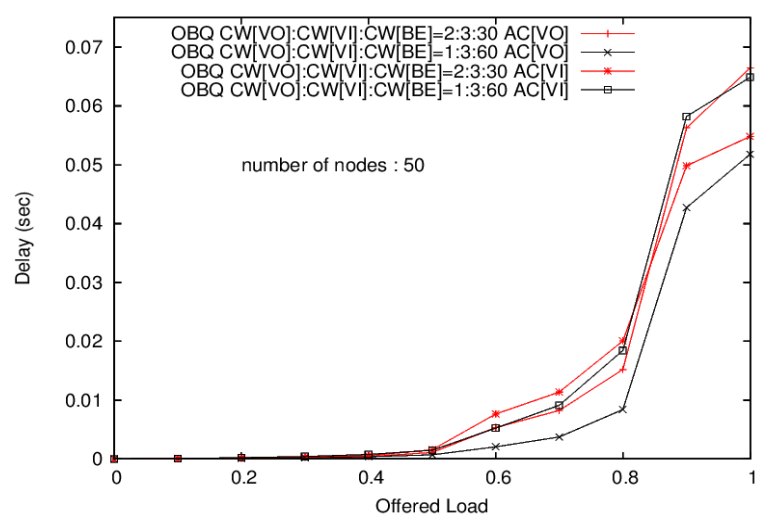

Fig. 9. Delay of $A C[V O]$ and $A C[V I]$ vs. offered traffic when the $C W$ ratio is changed.

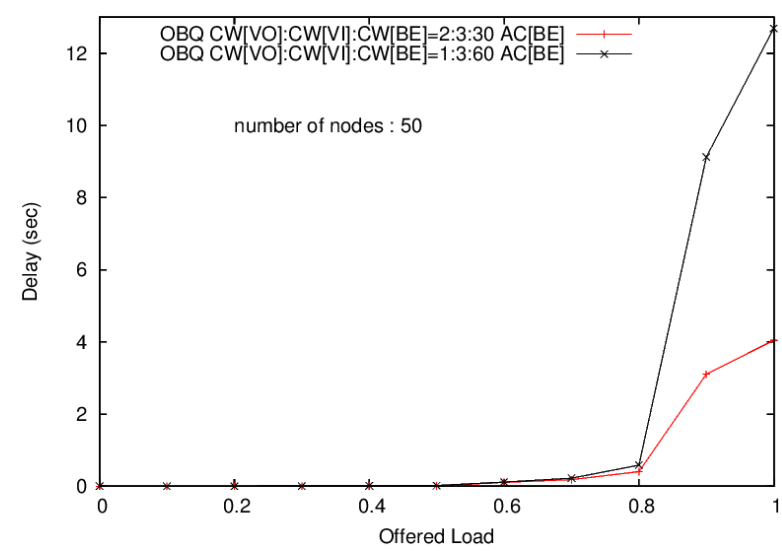

Fig. 10. Delay of $A C[B E]$ vs. offered traffic when the $C W$ ratio is changed.

\section{Data Dropped}

Fig. 11 shows the data dropped results when the number of nodes is 50 and the packet size is 1280 bytes. Packets are dropped due to buffer overflow and retry threshold exceeded. Buffer overflow is the number of the higher layer packets that are dropped because the node could not receive any ACK for the packets. Retry threshold exceeded is the number of the higher layer packets that are dropped because the node consistently fails retransmissions. In figure, vertical axis expresses the sum of buffer overflow and retry threshold exceeded and horizontal axis expresses normalized offered total traffic. As shown in Fig. 3-Fig. 5, OBQ maintains high throughput even if $C W$ ratio is changed. Therefore, also in case of the result of data dropped, minimize data dropped even if $C W$ ratio is changed. IEEE $802.11 \mathrm{e}$ is saturated from offered traffic 0.5 . In contrast, OBQ is not saturated when offered traffic 0.5 and is saturated from offered traffic 0.6.

It is found the reason that the delay of the IEEE 802.11e is lower than that of OBQ in saturated case. For the IEEE $802.11 \mathrm{e}$ has the $C W$ much lower than the optimal $C W$, the delay of IEEE 802.11e is lower than that of OBQ but IEEE 802.11 e occurred many data dropped. In other words, in case of lower delay, it is possible that can be sent to the destination early. However, IEEE 802.11e has extremely low guarantee at successful transmission for many data dropped and lower throughput. In contrast, OBQ minimizes data dropped moreover minimizes delay and obtains high throughput.

\section{E. Fairness}

To evaluate the fairness of OBQ, we adopt the following Fairness Index $(F I)[20]$ that is commonly accepted:

$$
F I=\frac{\left(\sum_{i=1} T_{i} / \phi_{i}\right)^{2}}{n \sum_{i=1}\left(T_{i} / \phi_{i}\right)^{2}}
$$

where $T_{i}$ is throughput of flow $i, \phi_{i}$ is the weight of flow $i$ (normalized throughput requested by each node). Here, we assume all nodes have the same weight in simulation. According to (8), $F I \leq 1$, where the equation holds only when all $T_{i} / \phi_{i}$ are equal. Normally, a higher FI means a better fairness.

Fig. 12 shows the fairness index of OBQ and the IEEE 802.11 e when packet size is 1280 bytes. It can be observed that the fairness of OBQ within 2s slightly decreases. OBQ can obtain better fairness than IEEE 802.11e even if $C W$ ratio is changed. This is because OBQ ensures that all the nodes use about the same $C W$ that is around the optimal value.

\section{F. Effect of Traffic Configuration}

Until now, The simulation parameter is that all nodes have three $A C \mathrm{~s}$, thus $A C[V O], A C[V I]$ and $A C[B E]$. However, in this section, the simulation parameter is that 25 active nodes have only $A C[V O]$ and 25 active nodes have only $A C[B E]$. Other simulation parameters are the same in the above section. Packet size is 1280 bytes and $C W$ ratio $C W[V O]: C W[V I]$ : $C W[B E]=2: 3: 30$. Fig. 13 shows the throughput results of each $A C$. In figure, vertical axis expresses normalized throughput of each $A C$ and horizontal axis expresses 
normalized offered total traffic. In case of IEEE 802.11e, the throughput of higher priority $A C[V O]$ is in saturated case from offered load 0.6 and decreases. Whereas for OBQ, the throughput of that always fulfills offered traffic. Improvement reaches to about $80 \%$ in offered load 1 . Also, throughputs of lower priority $A C[B E]$, both IEEE $802.11 \mathrm{e}$ and OBQ, decrease from a certain offered load. In case of IEEE 802.11e, throughput decrease sharply from offered load0.5. This is due to the reason that the variation of $C W$ on backoff in IEEE 802.11 e cannot adjust to the increased traffic of higher priority. OBQ always obtains high throughput of $A C[B E]$ and have less impact of the increased traffic, as opposed to IEEE 802.11 e.

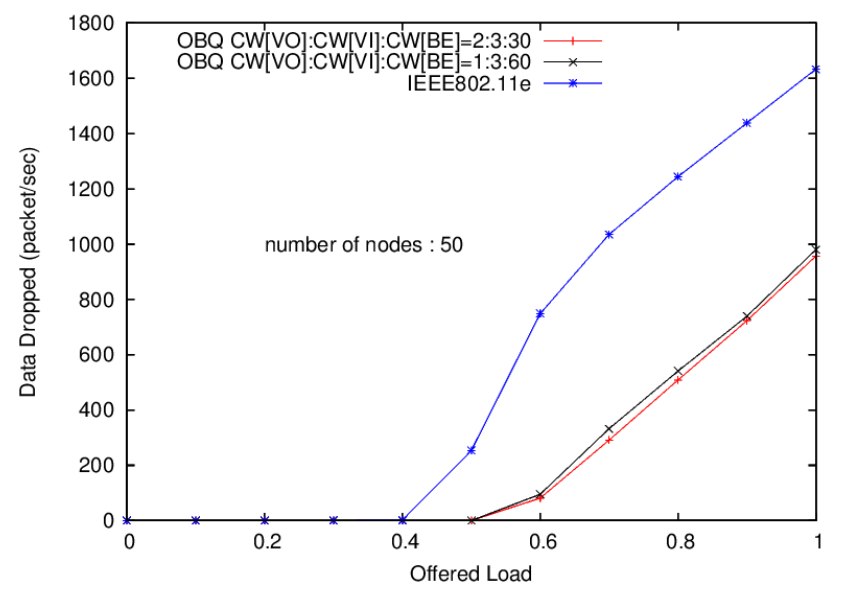

Fig. 11. Data dropped vs. offered traffic when the $C W$ ratio is changed.

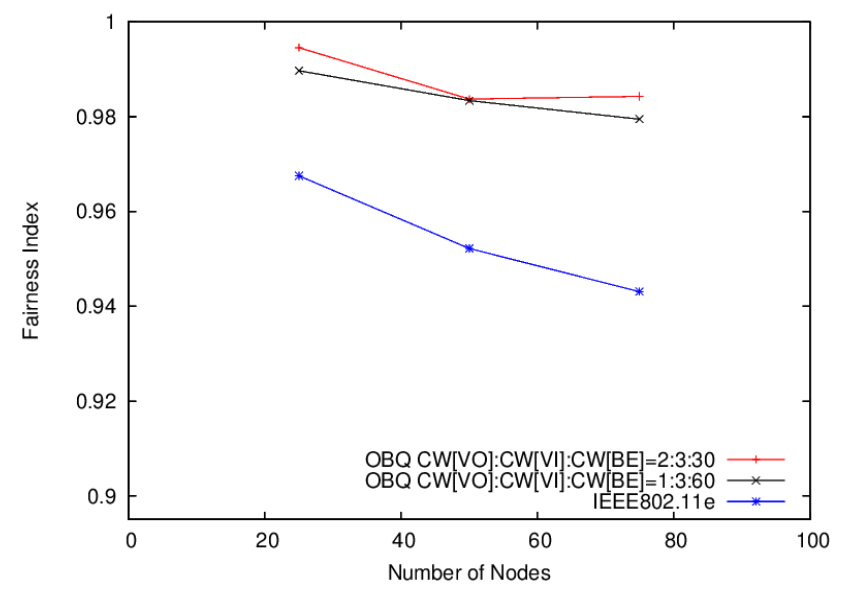

Fig. 12. Fairness index when the $C W$ ratio is changed.

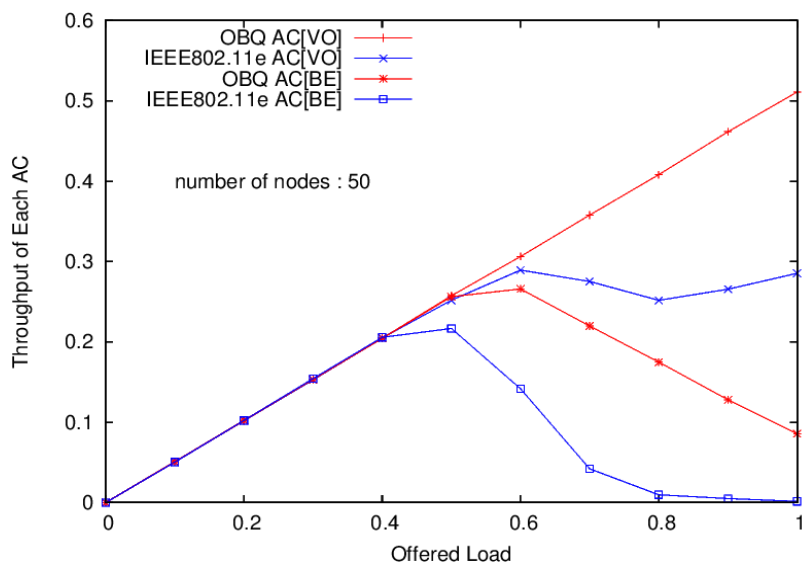

Fig. 13. Throughput of each $A C$ vs. offered traffic when the traffic configuration is changed.

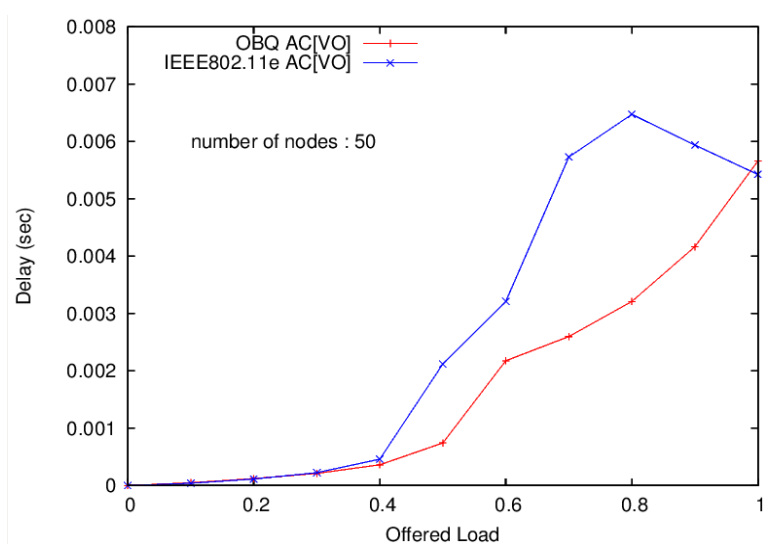

Fig. 14. Delay of $A C[V O]$ vs. offered traffic when the traffic configuration is changed.

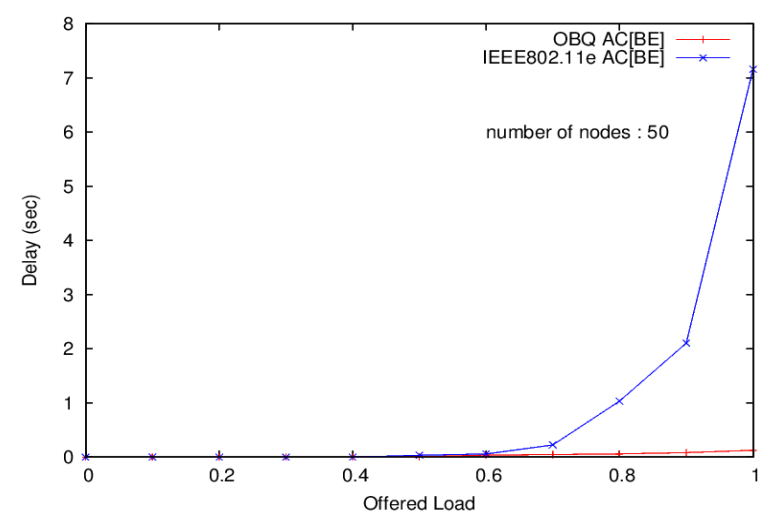

Fig. 15. Delay of $A C[B E]$ vs. offered traffic when the traffic configuration is changed.

Fig. 14 and Fig. 15 show the delay results of $A C[V O]$ and $A C[V I$, respectively. In Fig. 14, the delay of OBQ is lower than that of the IEEE 802.11e except offered load 1. The delay of OBQ is higher than that of the IEEE 802.11e in offered load 1, because we found the same reason that described in Section IV-C. In Fig. 15, the delay of OBQ is always lower than that of the IEEE 802.11e. Namely, the throughput of IEEE $802.11 \mathrm{e}$ decreases both $A C[V O]$ and $A C[B E]$ when traffic increases because the backoff algorithm of IEEE 802.11e cannot adjust effectively. In contrast, OBQ obtains high throughput and provide the satisfied QoS.

\section{CONCLUSION}

In this paper, we proposed a novel MAC protocol OBQ that enhance EDCA. In OBQ, each node observes three types of channel events, idle, successful transmission and collision to estimate the number of nodes and then sets optimal $C W$ dynamically according to the number of nodes. Thus, OBQ can obtain high throughput. With optimal $C W$ and $C W$ ratio according to the QoS requirement, each node sets $C W$ for each $A C$ separately, which leads to better QoS. Even if the traffics situation of each node changes, total throughput always maintains high throughput.

From analysis and simulation results, this scheme that observing channel and estimating the number of nodes is effective and adjust the network change promptly. Moreover, by using the optimal $C W$ according to the number of nodes and the $C W$ ratio according to the QoS requirement, OBQ solves the problems of conventional method and can achieve 
higher throughput and better QoS than IEEE 802.11e. All nodes with same traffic can have the almost same $C W$ around the optimal value, which means a high fairness. As a future work, we need verify by actual environment and evaluate the validity of OBQ.

\section{REFERENCES}

[1] F. Cali, M. Conti, and E. Gregori, "Dynamic tuning of the IEEE 802.11 protocol to achieve a theoretical throughput limit," IEEE/ACM Transactions on Networking, vol. 8, no. 6, pp. 785-799, Dec. 2000.

[2] B. Bianchi, "Performance analysis of the ieee 802.11 distributed coordination function," IEEE Journal on Selected Areas in Communications, vol. 18, no. 3, pp. 535-547, Mar. 2000.

[3] Y. C. Tay and K. C. Chua, "A capacity analysis for the IEEE 802.11 mac protocol," ACM/Baltzer Wireless Networks, vol. 7, pp. 159-171, Mar. 2001.

[4] J. H. Kim and J. K. Lee, "Performance of carrier sense multiple access with collision avoidance protocols in wireless LANs," Wireless Personal Communications, vol. 11, pp. 161-182, 1999.

[5] J. Weinmiller, H. Woesner, J. P. Ebert, and A. Wolisz, "Analyzing and tuning the distributed coordination function in the IEEE 802.11 dfwmac draft standard," MOSCOT, Feb. 1996.

[6] Y. Kwon, Y. Fang, and H. Latchman, "A novel mac protocol with fast collision resolution for wireless LANs," in Proc. IEEE INFOCOM 2003, Apr. 2003, vol. 2, pp. 853-862.

[7] M. Heusse, F. R. Guillier, and A. Duda, "Idle sense: an optimal access method for high throughput and fairness in rate diverse wireless LANs," in Proc. SIGCOMM 2005, Aug. 2005, pp. 121-132.

[8] P. Serrano, A. Banchs, P. Patras, and A. Azcorra, "Optimal configuration of 802.11e EDCA for real-time and data traffic," IEEE Transactions on Vehicular Technology, vol. 59, pp. 2511-2528, Jun. 2010.

[9] M. Ibrahim and S. Alouf, "Design and analysis of an adaptive backoff algorithm for IEEE 802.11 DCF mechanism," in Proc. NETWORKING 2006, May 2006, pp. 184-196.

[10] X. Tian, X. Chen, T. Ideguchi, and Y. Fang, "Improving throughput and fairness in WLANs through dynamically optimizing backoff," IEICE Trans. Commun., vol. E88-B, no. 11, pp. 4328-4338, 2005.

[11] S. Chun, D. Xianhua, L. Pingyuan, and Z. Han, "Adaptive access mechanism with optimal contention window based on node number estimation using multiple thresholds," IEEE Transactions on Wireless Communicaitons, vol. 11, no. 6, pp. 2046-2055, Jun. 2012.

[12] X. Tian, T. Sanada, T. Okuda, and T. Ideguchi, "A novel mac protocol of wireless LAN with high throughput and fairness," in Proc. 38th Annual IEEE Conference on Local Computer Networks, 2013, pp. 688-691.

[13] A. Hamidian and U. Koerner, "An enhancement to the IEEE 802.11e EDCA providing QoS guarantees," Telecommunication Systems, vol. 31, no. 2-3, pp. 195-212, Mar. 2006.

[14] B. J. Chang, Y. H. Liang, and J. W. Chu, "Performance analyses of high-efficiency EDCA for reducing contention collision and increasing throughput in QoS-based IEEE 802.11e wireless networks," Journal of Information Science and Engineering, 2010, pp. 1991-2007.

[15] Y. W. Lan, H. H. Yeh, J. C. Chen, and Z. T. Chou, "Performance enhancement of IEEE 802.11e EDCA by contention adaption," in Proc. Vehicular Technology Conference, 2005.

[16] Y. Zhang, L. Yang, Q. Wang, and R. Guo, "A dynamic queue management algorithm for IEEE 802.11e EDCA wireless LAN," Journal of Computational Information Systems, vol. 8, no. 11, 2012.

[17] S. Sehrawat, R. P. Bora, and D. Harihar, "Performance analysis of QoS supported by enhanced distributed channel access(EDCA) mechanism in IEEE 802.11e," IAENG International Journal of Computer Science, vol. 33, no. 1, 2007.

[18] Z. N. Kong, D. H. K. Tsang, B. Bensaou, and D. Gao, "Performance analysis of IEEE $802.11 \mathrm{e}$ contention-based channel access," IEEE Journal on Selected Areas in Communications, vol. 22, no. 10, pp. 2095-2106, Dec 2004.
[19] Opnet modeler. [Online]. Available: http://www.opnet.com

[20] D. M. Chiu and R. Jain, "Analysis of the increase and decrease algorithms for congestion avoidance in computer networks," Computer Networks and ISDN Systems, vol. 17, pp. 1-14, Feb 1989.

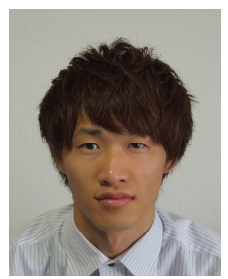

Takumi Sanada received the B.S degree form the Faculty of Information Science and Technology, Aichi Prefectural University in 2014.

$\mathrm{He}$ is now a graduate student in the School of Information Science and Technology, Aichi Prefectural University, Aichi, Japan.

His research interests include medium access control and QoS in wireless networks.

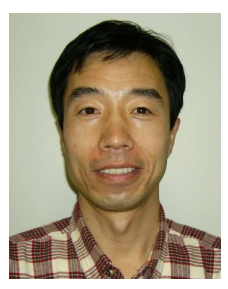

Xuejun Tian graduated from Hebei University, China in 1985. He received his M.S. degree from the Department of Electrical and Mechanical Engineering, Tianjin Institute of Technology, China in 1991, and the Ph.D. degree from the Department of Intelligence and Computer Science, Nagoya Institute of Technology, Japan, in 1998.

Since 1998, he had been an assistant professor and became an associate professor in 2008 at the Department of Information Systems, Faculty of Information Science and Technology, Aichi Prefectural University, Japan. From Jul. 2003 to Jun. 2004, he was a visiting assistant professor in the Department of Electrical and Computer Engineering at University of Florida, Gainesville, FL.

$\mathrm{He}$ is a member of IEICE (The Institute of Electronics, Information and Communication Engineers), IEEJ (the Institute of Electrical Engineers of Japan), IPSJ (Information Processing Society of Japan) respectively. His research interests include QoS, wireless networks, mobile communications and ubiquitous computing.

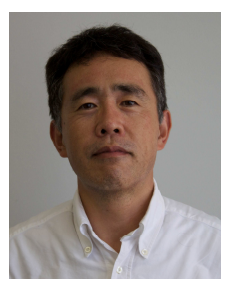

Takashi Okuda is a professor in the School of Information Science and Technology, Aichi Prefectural University, Nagakute, Japan. Dr. Takashi Okuda received the B.S, M.S, and Ph.D. degrees in engineering from Toyohashi University of Technology, Japan, in 1985, 1987, and 1992, respectively.

He joined Toyohashi University of Technology as a research associate and Asahi University as an associate professor in 1988 and 1993, respectively. He worked as a visiting professor at the Information Systems and Technologies Department, Weber State University, UT, from December 1994 to August 1995 and as a visiting scholar at the Computer Engineering Department, Duke University, NC, from July 2002 to January 2003.

His research interests include traffic engineering over communication networks, and information systems and technologies for business and society. He is a member of IEEE, IEICE, IPSJ, ORSJ, and JSET.

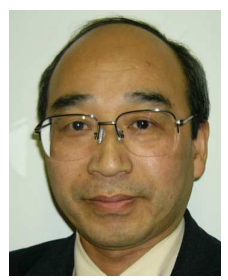

Tetsuo Ideguchi received the B.S. degree in telecommunication engineering from University of Electro-Communications in 1972, and the Ph.D. degree in telecommunication engineering from Tohoku University in 1993.

$\mathrm{He}$ is a professor at the Faculty of Information Science and Technology, Aichi Prefectural University, Aichi, Japan.

His interests include network architecture, LAN, network management and mobile communications. He is a member of IEEE, and IEICE and IPSJ in Japan. 